\title{
In emergencies, health research has to go beyond public engagement towards a true partnership with those affected
}

\begin{abstract}
An Ebola virus outbreak taking place in the complex political and social context of the DR Congo has forced the research community to reflect on their approach to community engagement. Katharine Wright and Michael Parker, on behalf of the Nuffield Council on Bioethics Working Group on the ethics of global health emergency research, say those affected need to influence research choices from the very beginning and the value of their knowledge must be recognised.
\end{abstract}

Katharine Wright, Michael Parker and the Nuffield Council on Bioethics Working Group

"When you don't see the problem the same way, you can't craft solutions together", said a West African social scientist who was drawing on decades of experience working with communities during disease outbreaks. He urged us to see beyond the procedural aspect of community engagement. We needed to see the artistry involved in "bringing two worlds faceto-face to build consensus".

This shift in thinking is a new perspective and one that is needed for the benefits of research to be realised - where research doesn't attribute a special status for one kind of expertise over another, but recognises the core contribution of different forms and sources of knowledge. For research to be built on relationships of equal and mutual respect, the voices of those affected need to influence research choices from the very beginning - not just 'engaged' once a study protocol is on the verge of being up and running, with the aim of maximising acceptability.

This finding underpins a groundbreaking report and Call for Action published on the $28^{\text {th }}$ January 2020 by the Nuffield Council on Bioethics. ${ }^{1}$ We recommend the following:

- Funders of emergency research must not initiate and support research unless the basic health needs of research participants are being met - for example through working in partnerships with humanitarian organizations and ministries of health.

- Funders and governments must invest in putting community engagement mechanisms into emergency research to make them a reality - for example, through funders explicitly including budget lines for community engagement and requiring feedback of research findings to participants when the research is over.

- Funders must do more to facilitate fair and equitable collaborations between research organisations - for example by funding scoping meetings between potential partners from high income and low income countries, and including budget lines for immediate capacity support for less well-resourced partners. In the longer-term funders should offer support for strengthening institutional research capacity.

The DR Congo - balancing the decision to trust against the fear of losing one's life

In recent months more and more people have been talking about the importance of community engagement and trust, in part in response to the public health and humanitarian 
response to the ongoing Ebola outbreak in the Democratic Republic of the Congo. In such emergencies, it is clear that equal and respectful relationships between the communities that are affected, the healthcare system (including national ministries of health) and research teams seeking to understand how to improve access to care and develop effective treatments, are paramount to the success of research.

The response in the DRC has been made enormously more dangerous and complex by its location in a conflict zone, where inhabitants have little reason to trust outsiders. Despite these challenges, it has seen a number of recent research successes, including the roll-out of an effective vaccine ${ }^{2}$ and the evaluation of two candidate treatments for Ebola virus disease. ${ }^{3}$

The gains made by research in this area were made possible through close collaboration between the national research institution and ministry of health, domestic and overseas research teams, and humanitarian organisations, with co-ordination and support through WHO. Critically, the national ministry of health took a leading role in setting the research strategy.

And yet, low levels of community trust have challenged efforts to control the outbreak and undermined the capability of research findings to change lives on the ground.

The ring vaccination strategy used with the VSV Ebola vaccine, for example, depends on vaccinating all the 'contacts of contacts' of those affected. This requires healthworkers working on the ground to gain the trust of communities to be able to identify those needing vaccination, and for people affected to consent to it. Trust is crucial here - offering effective treatments has little value if people affected by the virus do not trust the people nor locations offering treatment. All these challenges illustrate the need for a crucial re-think of approaches to research in such circumstances, to ensure that potentially valuable research findings can translate directly into positive benefit for those affected by the emergency.

Ebola is far from the most pressing concern for many people in affected areas - fear of sexual and other violence and of other diseases including measles and malaria, are much more immediate for many.

One DRC citizen said of the humanitarian health sector - "You will leave when Ebola does, but we will still be here, slowly dying from the diseases that have always killed us". ${ }^{4}$ Is it surprising that many people in the DRC are suspicious of the international community's motives, and some simply don't believe that Ebola is real ${ }^{5}$

\section{What needs to change? Value learned experience equally}

The international community has learned a lot in recent years about how best to integrate research into outbreak response. Considerable effort has gone into supporting community

2 Callaway (2019) Make Ebola a thing of the past': first vaccine against deadly virus approved Nature news Published online: 12 November. https://www.nature.com/articles/d41586-01903490-8.

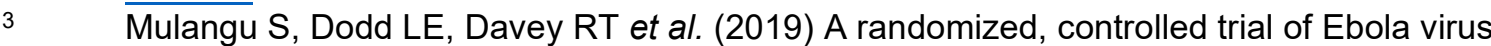
disease therapeutics New England Journal of Medicine: Published online: 27 November. https://www.niaid.nih.gov/news-events/independent-monitoring-board-recommends-earlytermination-ebola-therapeutics-trial-drc

$4 \quad$ Nguyen V-K (2019) An epidemic of suspicion - Ebola and violence in the DRC New England Journal of Medicine 380: 1298-9.

$5 \quad$ Vinck $P$ et al (2019) Institutional trust and misinformation in the response to the 2018-19 Ebola outbreak in North Kivu, DR Congo: a population-based survey The Lancet Infectious diseases 19(5): 529-536 
sensitisation and engagement, underpinned by social science research seeking to better understand the concerns of affected populations.

But how can health research hope to effectively reduce suffering if those allocating money to research projects and those designing the studies don't have a genuine understanding of the needs, concerns and priorities of those most affected?

Only by re-setting our most basic assumptions about whose knowledge is of value can we, as a research community, hope to achieve our (highly laudable) aims. Well-organised survivors' groups from previous outbreaks in the region can offer valuable insights when a disease like Ebola re-emerges even in the most remote and hard-to-reach areas.

There are also small but powerful ways in which the balance of power can be redressed. One is to be mindful of the narrative attached to the lack of community confidence in the research. The use of terms such as "population resistance", for instance, privileges the perspective of the responder over the nuanced concerns of diverse groups within local populations, and should be avoided.

'Engagement' is not enough. Trust has to be earned. We recognise that this new reality for research is a difficult ask, and one that can be very challenging in the midst an emergency. But difficulty must not be a barrier to action.

Achieving this goal will require funders, research institutions, research ethics committees and many others to challenge both their assumptions and their working methods. Their decision-making should now be centered around the priorities and needs of those they exist to support. It will also require supporting long-term capacity strengthening to boost the ability of national and local researchers to take the lead in responding to health emergencies in their countries.

Beyond the emergency situation, these findings have resonance in other health research settings. But like many extremes, the uncertainty and disruption of an emergency situation amplifies the problems that are encountered in less acute ways in other research, making them more visible and harder to ignore. 